\title{
Long-term outcomes in patients with benign central airway stenosis or obstruction following stenting
}

\author{
AKIRA IYODA $^{1}$, YOKO AZUMA ${ }^{1}$, ATSUSHI SANO ${ }^{1}$, TAKASHI SAKAI $^{1}$, SATOSHI KOEZUKA $^{1}$, \\ HAJIME OTSUKA ${ }^{1}$, KAZUTOSHI ISOBE ${ }^{2}$, SUSUMU SAKAMOTO ${ }^{2}$, YOSHINOBU HATA ${ }^{1}$ and KEIGO TAKAGI ${ }^{3}$ \\ ${ }^{1}$ Division of Chest Surgery, Department of Surgery, and ${ }^{2}$ Division of Respiratory \\ Medicine, Toho University School of Medicine, Tokyo 143-8541; ${ }^{3}$ Nishiyokohama \\ International Hospital, Yokohama, Kanagawa 245-8560, Japan
}

Received May 27, 2020; Accepted July 15, 2020

DOI: $10.3892 /$ wasj.2020.61

\begin{abstract}
Although an airway stent is used for central airway stenosis or obstruction due to benign diseases, the long-term outcomes of stenting are unknown. The aim of the present study was to identify the clinical features and outcomes of patients with airway stenosis or obstruction due to benign disease who underwent stenting procedures. For this purpose, clinical data from 136 patients with stents for central airway stenosis or obstruction who underwent treatment at Toho University Omori Medical Center from 1998 to 2018 were retrospectively analyzed. The clinical features, outcomes and complications associated with stenting were evaluated. The clinical features of patients with stents for benign central airway stenosis or obstruction were compared to those of patients with stents for malignant disease. Of the 136 patients with stents, 19 patients had benign disease and 117 had malignant disease. The difference between complications in patients with benign disease compared with those with malignant disease was not significant. The median follow-up time of patients with stents due to benign central airway stenosis or obstruction was 51 (range, 0.164-239) months. Patients with benign disease had a significantly better outcome than patients with malignant disease. For the 19 patients with benign disease, 35 stent procedures were performed as follows: A total of 18 placements, 6 replacements, 3 additional stent placements, 6 removals and 2 stents were repositioned. Patients with benign disease underwent significantly more $(\geq 2)$ stent-related procedures than patients with malignant disease. On the whole, the findings of the present study indicate that patients with benign central airway stenosis or obstruction who undergo stenting may require multiple procedures, and that physicians should plan for multiple treatments following the first stent placement as patients with benign airway disease have good long-term outcomes.
\end{abstract}

Correspondence to: Professor Akira Iyoda, Division of Chest Surgery, Department of Surgery, Toho University School of Medicine, 6-11-1 Omori-Nishi, Ota-ku, Tokyo 143-8541, Japan

E-mail: aiyoda@med.toho-u.ac.jp

Key words: airway, stent, airway stenosis, obstruction, benign

\section{Introduction}

In patients with benign central airway stenosis or obstruction, surgical resection/anastomosis is the optimal treatment: However, only $50 \%$ of patients can undergo resection (1). Therefore, stenting is required for the treatment of patients with benign airway stenosis or obstruction (1). An airway stent is used to improve symptoms and quality of life. In addition, airway stenting is used as palliative treatment to improve lung function in patients with central airway stenosis or obstruction due to thoracic malignancies (2-4), as central airway stenosis or obstruction can result from tumor invasion or extrinsic tumor compression $(5,6)$. However, the decision for stent placement in an airway should be undertaken carefully, as stenting is associated with serious risks, such as bleeding, airway obstruction during the procedure, or misplacement or migration from the desired location (5). In particular, care should be taken in selecting the types of stenting according to the indications for patients with benign central airway stenosis or obstruction (5). Therefore, the indications for stent placement in patients with benign disease should be based on a risk-benefit analysis.

Reports on stenting for airway stenosis or obstruction due to malignant disease are relatively common; however, limited data are available on stenting for patients with central airway stenosis or obstruction due to benign disease, as there are only a few studies available on the long-term outcomes of patients with benign disease who have undergone airway stenting $(1,7)$. As the outcomes for patients with benign disease may differ from those of patients with malignant disease (7), and a number of issues related to stenting for benign central airway stenosis or obstruction remain unknown (8), the accrual of data on stenting related to benign disease is warranted. The aim of the present study was to identify the clinical features and outcomes of patients with airway stenosis or obstruction due to benign disease who underwent stenting procedures.

\section{Materials and methods}

Patients. The present study was approved by the Ethics Committee of Toho University Omori Medical Center (M19151). The present study retrospectively analyzed clinical data from 19 patients with stents for benign airway stenosis or obstruction 
Table I. Characteristics of patients with airway stenosis/obstruction.

\begin{tabular}{|c|c|c|c|c|}
\hline Clinical factor & $\begin{array}{c}\text { Total no. } \\
\text { of patients } \\
136\end{array}$ & $\begin{array}{c}\text { No. of patients } \\
\text { with benign disease } \\
19\end{array}$ & $\begin{array}{c}\text { No. of patients with } \\
\text { malignant disease } \\
117\end{array}$ & P-value \\
\hline \multicolumn{5}{|l|}{ Age } \\
\hline Mean & 63 & 57 & 64 & 0.4817 \\
\hline \multicolumn{5}{|l|}{ Sex } \\
\hline Male:female & $94: 42$ & $8: 11$ & $86: 31$ & 0.0131 \\
\hline Type of stent & & & & 0.2915 \\
\hline Silicon & 60 & 11 & 49 & \\
\hline Metallic & 76 & 8 & 68 & \\
\hline Treatment & 167 & 35 & 132 & $<0.0001$ \\
\hline Placement & 135 & 18 & 117 & \\
\hline Replacement & 10 & 6 & 4 & \\
\hline $\begin{array}{l}\text { Additional } \\
\text { placement }\end{array}$ & 9 & 3 & 6 & \\
\hline Removed & 9 & 6 & 3 & \\
\hline Repositioned & 3 & 2 & 1 & \\
\hline Cleaned & 1 & 0 & 1 & \\
\hline
\end{tabular}

who underwent treatment at Toho University Omori Medical Center from 1998 to 2018, and compared the data with those of patients with stents for central airway stenosis or obstruction due to malignant disease. The following parameters were investigated from the medical records obtained from Toho University Omori Medical Center: Sex, age, disease, types of stents, complications, treatment after stenting and patient outcome.

Complications associated with stenting. The following complications were evaluated as positive if treatment via bronchoscopy was required: Granulations, halitosis, infection, mucoid impaction, tumor ingrowth, stent fracture, stent malposition, or stent migration. Bleeding caused by stenting was considered positive if hemostatic agents were administered or bronchoscopic treatment was required. Infections, stent fracture and airway rupture were considered related to stenting if confirmed by bronchoscopy. Halitosis was considered positive if patients did not have halitosis prior to stenting, but developed it after stenting.

Outcomes. The outcomes of patients following stenting for airway stenosis or obstruction due to benign or malignant disease were compared.

Statistical analysis. Fisher's exact test was used to compare binomial proportions in frequency of complication by type. The $\chi^{2}$ test was used to assess differences between sex, type of stent, treatments in patients' characteristics, complications of stenting, and cases undergoing $\geq 2$ procedures. Mann-Whitney $\mathrm{U}$ test was used to assess differences in age in the patient characteristics. Survival times were measured from the date of the first stent treatment until the date of death or the last follow-up, and were estimated by the Kaplan-Meier method. Kaplan-Meier graphs were compared by the log-rank test. A P-value $<0.05$ was considered to indicate a statistically significant difference.
Table II. Reasons for stenting in patients with benign disease.

\begin{tabular}{lc}
\hline Group & No. of patients \\
\hline Benign disease & 19 \\
After intubation & 8 \\
After tracheostomy & 7 \\
Traffic accident & 1 \\
After surgery for infection & 1 \\
Infection (tuberculosis) & 1 \\
Goiter & 1 \\
\hline
\end{tabular}

\section{Results}

The patient characteristics are presented in Table I. Of the patients with benign disease, a significantly higher proportion were females, whereas this was not the case in patients with malignant disease (Table I). The difference between the types of stents (silicon vs. metallic) used in patients with benign vs. those with malignant disease was not significant.

Of the 19 patients with benign disease, central airway stenosis or obstruction due to long-term intubation, tracheostomy, traffic accident, surgery for infection, tuberculosis and goiter resulted in stenting for 8 patients, 7 patients, 1, 1, 1 and 1 patient, respectively (Table II). The differences between complication rates and types of complication in patients with benign disease vs. those with malignant disease were not significant (Tables III and IV).

The median follow-up times of patients with stents due to benign and malignant central airway stenosis or obstruction were 51 (benign: Range, 0.164-239,) months and 3 (malignant: Range, 0.066-125) months, respectively. The 5-year survival rates were 80.5 and $6.3 \%$ in patients with benign and 
Table III. Complications of stenting.

\begin{tabular}{lccc}
\hline Group & $\begin{array}{c}\text { Total no. } \\
\text { of patients }\end{array}$ & $\begin{array}{c}\text { No. of patients } \\
\text { with benign disease }\end{array}$ & $\begin{array}{c}\text { No. of patients } \\
\text { with malignant disease }\end{array}$ \\
\hline Positive for complication & 33 & 6 & 27 \\
Negative for complication & 103 & 13 & 90 \\
\hline
\end{tabular}

Table IV. Frequency of complication by type.

\begin{tabular}{lcccr}
\hline Group & $\begin{array}{c}\text { Total no. } \\
\text { of patients }\end{array}$ & $\begin{array}{c}\text { No. of patients } \\
\text { with benign disease }\end{array}$ & $\begin{array}{c}\text { No. of patients with } \\
\text { malignant disease }\end{array}$ & P-value \\
\hline Airway rupture & 0 & 0 & 0 & - \\
Bleeding & 2 & 0 & 2 & $>0.9999$ \\
Granulation & 9 & 2 & 7 & 0.6129 \\
Halitosis & 3 & 1 & 2 & 0.3656 \\
Infection & 3 & 0 & 3 & $>0.9999$ \\
Mucoid impaction & 14 & 2 & 12 & $>0.9999$ \\
Stent fracture & 0 & 0 & 0 & - \\
Stent malposition & 2 & 1 & 1 & 0.2608 \\
Stent migration & 11 & 3 & 8 & 0.1837 \\
Total & 44 & 9 & 35 & \\
\hline
\end{tabular}

malignant stenosis or obstruction, respectively. The 10 -year survival rates were 58.7 and $3.1 \%$ in patients with benign and malignant stenosis or obstruction, respectively. Patients with benign disease had a significantly better outcome than patients with malignant disease (Fig. 1, $\mathrm{P}<0.0001$ ).

For 19 patients with benign disease, 35 stent procedures were performed, which consisted of the following: A total of 18 placements, 6 replacements, 3 additional stent placements, 6 removals and 2 stents repositioned. One patient underwent removal and replacement of the silicon stent in Toho University Omori Medical Center following the placement of the silicon stent in another hospital (Table V, case 4). The differences between the types of treatments for patients with benign disease vs. those with malignant disease were significant (Table I, $\mathrm{P}<0.0001$ ). As regards the number of procedures, 7 out of 19 patients with benign disease had $\geq 2$ procedures and 13 out of 117 patients with malignant disease had $\geq 2$ procedures; in addition, 6 out of 19 patients with benign disease had $\geq 3$ procedures, and only 2 of the 117 patients with malignant disease had $\geq 3$ procedures (Table V). Patients with benign disease underwent significantly higher numbers of procedures ( $\geq 2$ and $\geq 3$ ) than patients with malignant disease (Table VI; $\mathrm{P}=0.0096$ and $\mathrm{P}<0.0001$, respectively).

\section{Discussion}

The findings of the present study indicated that patients with benign disease obtained significantly better outcomes after airway stenting than patients with malignant disease, and patients with benign disease had significantly more frequent operations than patients with malignant disease. These results

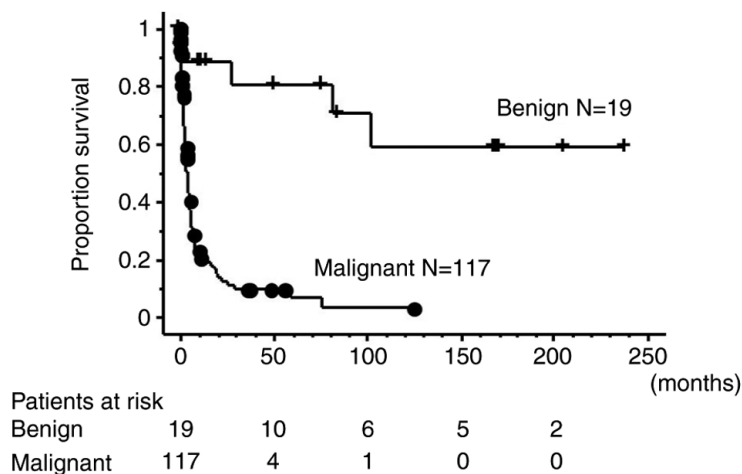

Figure 1. Overall survival curves of 136 patients undergoing stenting for benign central airway stenosis or obstruction and for patients with malignant disease. Patients with benign disease had a significantly better outcome than patients with malignant disease $(\mathrm{P}<0.0001)$.

indicate that patients with benign central airway stenosis or obstruction who undergo stenting might require multiple procedures, and that physicians should plan for multiple treatments following the first stent placement due to their promising outcomes. Although retrospective studies on airway stenting have revealed risks and complications after stenting $(5,6)$, as there are minimal data available on long-term outcomes, the present study investigated the clinical features and outcomes of patients with airway stenosis or obstruction due to benign disease.

Previous studies on airway stenting for benign central airway obstruction have been retrospective and have included few patients and short follow-up periods, as prospective 
Table V. Cases undergoing $\geq 2$ procedures.

\begin{tabular}{|c|c|c|c|c|c|c|}
\hline Group & Reason for obstruction & 1st operation & 2nd operation & 3rd operation & 4th operation & 5 th operation \\
\hline \multicolumn{7}{|c|}{ Benign disease } \\
\hline Case 1 & Traffic accident & Ultraflex & Dumon(ad) & Dumon(re) & & \\
\hline Case 2 & Tracheostomy & Dumon & Removal & & & \\
\hline Case 3 & Infection & Ultraflex & Ultraflex(ad) & Dumon(ad) & & \\
\hline Case 4 & Tracheostomy & [Dumon] & Removal & Dumon(re) & & \\
\hline Case 5 & Intubation & Dumon & Repositioned & Dumon(re) & Removal & \\
\hline Case 6 & Tracheostomy & Dumon & Removal & Dumon(re) & Dumon(re) & \\
\hline Case 7 & Tracheostomy & Dumon & Repositioned & Removal & T-tube(re) & Removal \\
\hline \multicolumn{7}{|c|}{ Malignant disease } \\
\hline Case 1 & Thyroid cancer & Dumon & Dumon(re) & & & \\
\hline Case 2 & Lymphoma & Dumon & Ultraflex(ad) & & & \\
\hline Case 3 & Lung cancer & Ultraflex & Ultraflex(ad) & & & \\
\hline Case 4 & Lung cancer & Dumon & Removal & Ultraflex(re) & & \\
\hline Case 5 & Thyroid cancer & Ultraflex & Ultraflex(ad) & T-tube(ad) & & \\
\hline Case 6 & Lung cancer & Dumon & Removal & & & \\
\hline Case 7 & Lung cancer & Ultraflex & Ultraflex(ad) & & & \\
\hline Case 8 & Thyroid cancer & Ultraflex & Cleaned & & & \\
\hline Case 9 & Esophageal cancer & Ultraflex & Ultraflex(ad) & & & \\
\hline Case 10 & Metastatic cancer & Dumon & Removal & & & \\
\hline Case 11 & Malignant schwannoma & Dumon & Ultraflex(re) & & & \\
\hline Case 12 & Lung cancer & Dumon & Repositioned & & & \\
\hline Case 13 & Esophageal cancer & Dumon & Dumon(re) & & & \\
\hline
\end{tabular}

For benign case 4 square brackets ' [ ]' indicate that this procedure was performed in other hospital. (ad), additional placement; (re), replacement. Dumon, Dumon stent; Ultraflex, Ultraflex stent.

Table VI. Differences between patients with benign and malignant disease undergoing $\geq 2$ and $\geq 3$ procedures.

\begin{tabular}{lcrr}
\hline No. of procedures & Benign disease $(\mathrm{n}=19)$ & Malignant disease $(\mathrm{n}=117)$ & $\mathrm{P}-\mathrm{value}$ \\
\hline Patients with $\geq 2$ procedures & 7 & 13 & 0.0096 \\
Patients with $\geq 3$ procedures & 6 & 2 & $<0.0001$ \\
\hline
\end{tabular}

and large-scale studies were difficult to perform for airway stenting $(1,5)$. Saad et al examined the clinical features of 21 patients with miscellaneous benign diseases and 11 patients with airway complications following lung transplantation (7). They reported median follow-up durations of 336 days for patients with miscellaneous benign diseases and 329 days for patients following lung transplantation. Husain et al performed a retrospective study on the Ultraflex metallic stent, and compared patients with benign airway obstruction with patients with malignant airway obstruction (1). They reported that 10 of 12 patients with benign airway obstruction were alive with a median survival of 1,017 days, ranging from 46 to 1,120 days, but not reaching the 5-year endpoint. Of the 2 patients with benign diseases who passed away, 1 had severe Wegener granulomatosis and the other had the comorbidities of pulmonary fibrosis and cardiac failure (1).

By contrast, Saji et al examined 65 patients with advanced lung cancer associated with central airway obstruction and found a 1-year survival rate of $25.2 \%$ and a median survival time of 6.2 months (9). Furukawa et al examined 40 patients with advanced lung cancer associated with central airway obstruction and found a 1-year survival rate of $14.3 \%$ and median survival time of 94 days (3.1 months) (10). Lemaire et al performed a retrospective study on 140 patients with malignant tumor and malignant airway disease and found a 1-year survival rate of $15 \%$ and median survival of 3.4 months after stenting (11).

In 2005, the US Food and Drug Administration recommended that metallic stents should be avoided for benign central airway obstruction (5). Although wire stents preserve the mucociliary escalator, permit a larger airway lumen, and are more easily implanted than other stents, some investigators have advised that wire stents should not be used as patients with metallic stents placed for benign tracheobronchial disease have a significantly higher complication rate than patients with metallic stents placed for malignancy (12-14). Wire stents have 
also been found to be difficult to remove after they have been in the patients for a long period of time (1). On the other hand, silicon stents have such disadvantages as migration, small internal lumens and impairment of the mucociliary escalator, although they have been easy to remove (1). Although in the present study, no significant differences were found in the complications between silicon and metallic stents, long-term survival and possible multiple stent-related procedures need to be considered for patients with benign airway stenosis or obstruction.

The present study has certain limitations. First, this was a retrospective study. Second, the present study included a small number of patients with benign central airway stenosis or obstruction. However, despite these limitations, the findings were significant.

In conclusion, the present study found that the majority of patients with benign central airway stenosis or obstruction needed multiple stent-related procedures as the patients had better outcomes than patients with malignant disease. Therefore, airway stenting for patients with benign central airway obstruction should be performed, while considering the need for $>1$ stent-related procedure.

\section{Acknowledgements}

Not applicable.

\section{Funding}

No funding was received.

\section{Availability of data and materials}

All data generated or analyzed during this study are included in this published article.

\section{Authors' contributions}

AI, YA, AS, TS, SK, HO, KI, SS, YH and KT were involved in the conception and design of the study. AI wrote the manuscript. YA, AS, TS, SK, HO, KI, SS and YH collected data. KT supervised the study. All authors have read and approved the final manuscript.

\section{Ethics approval and consent to participate}

The present retrospective study was approved by the Ethics Committee of Toho University Omori Medical Center (M19151).

\section{Patient consent for publication}

Not applicable.

\section{Competing interests}

The authors declare that they have no competing interests.

\section{References}

1. Husain SA, Finch D, Ahmed M, Morgan A and Hetzel MR: Long-term follow-up of ultraflex metallic stents in benign and malignant central airway obstruction. Ann Thorac Surg 83: 1251-1256, 2007.

2. Oviatt PL, Stather DR, Michaud G, Maceachern P and Tremblay A: Exercise capacity, lung function, and quality of life after interventional bronchoscopy. J Thorac Oncol 6: 38-42, 2011.

3. Ferrell B, Koczywas M, Grannis F and Harrington A: Palliative care in lung cancer. Surg Clin North Am 91: 403-417, 2011

4. Sökücü SN, Özdemir C, Tural Önür S, Dalar L and Altın S: Comparison of silicon and metallic bifurcated stents in patients with malignant airway lesions. Clin Respir J 14: 198-204, 2020.

5. Folch E and Keyes C: Airway stents. Ann Cardiothorac Surg 7: 273-283, 2018.

6. Ost DE, Shah AM, Lei X, Godoy MCB, Jimenez CA, Eapen GA, Jani P, Larson AJ, Sarkiss MG and Morice RC: Respiratory infections increase the risk of granulation tissue formation following airway stenting in patients with malignant airway obstruction. Chest 141: 1473-1481, 2012.

7. SaadCP, Murthy S, Krizmanich G and Mehta AC: Self-expandable metallic airway stents and flexible bronchoscopy: Long-term outcomes analysis. Chest 124: 1993-1999, 2003.

8. Murgu SD, Egressy K, Laxmanan B, Doblare G, Ortiz-Comino R and Hogarth DK: Central airway obstruction: Benign strictures, tracheobronchomalacia, and malignancy-related obstruction. Chest 150: 426-441, 2016.

9. Saji H, Furukawa K, Tsutsui H, Tsuboi M, Ichinose S, Usuda J, Ohira T and Ikeda N: Outcomes of airway stenting for advanced lung cancer with central airway obstruction. Interact Cardiovase Thorac Surg 11: 425-428, 2010.

10. Furukawa K, Ishida J, Yamaguchi G, Usuda J, Tsutsui H, Saito M, Konaka $\mathrm{C}$ and Kato $\mathrm{H}$ : The role of airway stent placement in the management of tracheobronchial stenosis caused by inoperable advanced lung cancer. Surg Today 40: 315-320, 2010.

11. Lemaire A, Burfeind WR, Toloza E, Balderson S, Petersen RP, Harpole DH Jr and D'Amico TA: Outcomes of tracheobronchial stents in patients with malignant airway disease. Ann Thorac Surg 80: 434-438, 2005.

12. Chung FT, Chen HC, Chou CL, Yu CT, Kuo CH, Kuo HP and Lin SM: An outcome analysis of self-expandable metallic stents in central airway obstruction: A cohort study. J Cardiothorac Surg 6: 46, 2011 .

13. Guibert N, Saka H and Dutau H: Airway stenting: Technological advancements and its role in interventional pulmonology. Respirology: Mar 11, 2020 doi: 10.1111/resp.13801 (Epub ahead of print).

14. Serrano C, Laborda A, Lozano JM, Caballero H, Sebastián A, Lopera $\mathbf{J}$ and de Gregorio MÁ: Metallic stents for tracheobronchial pathology treatment. Cardiovasc Intervent Radiol 36: 1614-1623, 2013 